\title{
Tratamiento de agresores contra la pareja en programas de atención a drogodependientes: un reto de futuro
}

\section{Addressing intimate partner violence in substance-abuse treatment programmes: a challenge for the future}

| JaVier Fernández-Montalvo*; José JaVier López-Goñi*; Alfonso ArteAGA*

\section{RESUMEN}

Existe una relación muy estrecha entre el consumo de sustancias (alcohol y otras drogas) y la violencia hacia la pareja. Los estudios llevados a cabo con hombres maltratadores y con pacientes adictos muestran una alta comorbilidad entre ambos fenómenos. Sin embargo, hasta la fecha han sido muy escasos los programas de intervención con maltratadores que se han aplicado en el ámbito de las drogodependencias. En este artículo se propone, en primer lugar, la necesidad de detectar los casos de violencia contra la pareja que aparecen camuflados bajo un problema de consumo de drogas. En este sentido, es necesario llevar a cabo estudios que determinen la tasa de prevalencia de agresores contra la pareja entre los usuarios de los programas de tratamiento para el abuso de sustancias, así como conocer las características específicas de este tipo de pacientes. En segundo lugar, una vez detectados los agresores, se podrían desarrollar programas específicos para el tratamiento simultáneo de ambas problemáticas (adicción y violencia hacia la pareja). Se han Ilevado a cabo algunos estudios con tratamientos conjuntos de la adicción y de la violencia de pareja. Los resultados obtenidos son esperanzadores y muestran que los programas de intervención con adicciones pueden ser un marco útil para aplicar también, en los casos necesarios, un tratamiento específico para aquellos adictos con un problema asociado de violencia contra la pareja. Se comentan las implicaciones de todo ello para la práctica clínica y para las investigaciones futuras.

Palabras Clave: adicciones, violencia contra la pareja, maltratadores, tratamiento
* Departamento de Psicología y Pedagogía. Universidad Pública de Navarra. Campus de Arrosadía. 31006 Pamplona.

Enviar correspondencia a:

Javier Fernández-Montalvo

Departamento de Psicología y Pedagogía

Universidad Pública de Navarra

Campus de Arrosadía

31006 Pamplona.

Teléfono: 948169830

Fax: 948169891

Correo electrónico: fernandez.montalvo@unavarra.es

\section{ABSTRACT}

There is a close relationship between substance abuse (alcohol and other drugs) and intimate partner violence. Studies carried out with male offenders and with addicted patients show a high comorbidity rate between these two phenomena. However, few batterer intervention programmes have been implemented to date in the field of drug addiction. This paper proposes, first, the need to detect cases of intimate partner violence that are camouflaged beneath a drug problem. Thus, it is important to determine the prevalence rate of intimate partner aggressors among users of drug-addiction treatment programmes, as well as identifying the specific characteristics of these patients. Second, once aggressors are identified, it would be possible to develop specific programmes for the simultaneous treatment of the two problems (addiction and intimate partner violence). Some studies have already been carried with joint treatments for addiction and intimate partner violence. The results obtained are encouraging, and show that intervention programs with addictions can be a useful framework for applying also, where necessary, specific treatments for those addicted patients with an associated problem of intimate partner violence. Finally, implications for clinical practice and future research in this field are discussed.

Key words: addictions, intimate partner violence, batterers, treatment 


\section{INTRODUCCIÓN}

L a relación entre el maltrato a la mujer y el consumo de sustancias por parte del agresor es un tema polémico de gran actualidad. El código penal vigente considera el consumo de alcohol como un atenuante en las penas impuestas por delitos de violencia de género (artículos 20 y 21 del Código Penal). En los últimos meses se han alzado numerosas voces críticas ante este hecho, principalmente desde asociaciones de víctimas e, incluso, desde medios judiciales. Todo ello ha provocado un debate intenso en los medios de comunicación, así como en el ámbito de los profesionales de la psicología que se dedican a temas de violencia de género.

Al margen de la polémica legal sobre la consideración del consumo de sustancias -en este caso el alcohol- como atenuante o agravante, la presencia del consumo de drogas y de alcohol entre las personas violentas en general y, más específicamente, entre los agresores a la mujer, está bien documentada. Los estudios sobre el perfil psicológico de los agresores, tanto nacionales como internacionales, coinciden en sus resultados. La tasa de agresores que presentan problemas de abuso o dependencia alcohólica puede oscilar entre el 50\% y el 60\% (Echeburúa, Fernández-Montalvo, y Amor, 2003; Fernández-Montalvo, Echeburúa, y Amor, 2005; Fernández-Montalvo y Echeburúa, 1997, 2005; Klostermann y Fals-Stewart, 2006; Stuart, 0'Farrell, y Temple, 2009). En el caso de otro tipo de drogas ilegales, los datos varían en las diferentes investigaciones pero, como media, en torno al $20 \%$ de los hombres agresores presenta un consumo abusivo de drogas (Fals-Stewart, Golden, y Schumacher, 2003; Fernández-Montalvo y Echeburúa, 1997, 2005; Moore et al., 2008; Stuart et al., 2008). En este sentido, la mayor parte de los estudios se han centrado en el consumo de cocaína y de cannabis. Los resultados específicos indican una fuerte asociación entre el consumo de estas drogas y la gravedad de los episodios violentos (Stuart, et al., 2008).

Esta misma relación se observa también cuando se analiza el problema desde la perspectiva de las drogodependencias. Aproximadamente entre un $40 \%$ y un $60 \%$ de los pacientes adictos que viven con sus parejas, presentan episodios violentos contra las mismas durante el año previo al inicio del tratamiento para su adicción (Easton, Swan, y Sinha, 2000; Fals-Stewart, et al., 2003; 0'Farrell y Murphy, 1995). Unos resultados similares se han observado incluso en pacientes que presentan problemas relacionados con la adicción al juego (Korman et al., 2008). Además, en algunos estudios ha quedado clara la influencia del consumo de sustancias, principalmente alcohol, en el desarrollo y desencadenamiento de episodios violentos contra la pareja (Fals-Stewart, 2003; Fals-Stewart, Leonard, y Birchler, 2005; Murphy, O'Farrell, Fals-Stewart, y Feehan, 2001; MuñozRivas, Gámez, Graña y Fernández, 2010; Stuart et al., 2009). Sin embargo, a pesar de la relación existente entre el consumo de sustancias y la presencia de conductas violentas, hoy por hoy no hay ningún estudio que establezca una relación causal entre ambos fenómenos (Leonard, 2005). Probablemente, el abuso de sustancias constituye un factor preci- pitante, más que un factor causal, de la violencia contra la pareja.

En cualquier caso, con arreglo a los datos encontrados, una línea urgente de trabajo para los próximos años debe centrarse en estudiar la relación entre el consumo de sustancias y la violencia contra la pareja, así como en establecer posibles vías específicas de intervención conjunta para ambas problemáticas (Fals-Stewart y Kennedy, 2005; Moore, et al., 2008; Stuart et al., 2009). Los programas de tratamiento de drogodependencias son sin duda un contexto adecuado para identificar situaciones de violencia de género.

\section{VIOLENCIA Y TRATAMIENTO DE LAS DROGODE- PENDENCIAS}

Debido a la estrecha relación entre violencia y adicciones, algunos autores han analizado la evolución de las conductas violentas entre los pacientes adictos que reciben tratamiento. Los resultados han puesto de manifiesto que la intervención estándar para las adicciones, en pacientes drogodependientes con problemas asociados de violencia contra la pareja, provoca una disminución importante en las conductas violentas, a pesar de no intervenir específicamente en este ámbito (O'Farrell, Fals-Stewart, Murphy, y Murphy, 2003; Stuart et al., 2003). En el estudio de O'Farrell et al. (2003), por ejemplo, con una muestra de 301 hombres alcohólicos, la presencia de violencia contra la pareja disminuyó del 56\% antes del tratamiento al 25\% después del mismo, a pesar de no haber recibido una intervención específica para ello. Algo similar ocurre en el estudio de Stuart et al. (2003), llevado a cabo con 24 pacientes en tratamiento por alcoholismo. Todos ellos presentaron disminuciones significativas en la frecuencia de las conductas violentas contra la pareja tras recibir un tratamiento para la adicción. En general, los distintos estudios llevados a cabo en este sentido muestran resultados similares. Una revisión más completa sobre este tema especifico, puede encontrase en Stuart et al., (2009). Además, en algunos estudios se ha observado que los pacientes alcohólicos que recaen no reducen las conductas violentas, mientras que aquellos que mantienen la abstinencia, mantienen también la reducción de las conductas violentas (0'Farrell y Murphy, 1995). Sin embargo, si bien la reducción o eliminación del consumo de alcohol o de otras drogas es necesaria para controlar las conductas violentas, probablemente sea insuficiente para la eliminación definitiva de las mismas.

\section{Programas específicos de tratamiento en el ámbi- to de las drogodependencias}

Hasta la fecha han sido muy escasos los esfuerzos desarrollados para el tratamiento de la violencia de pareja en el ámbito de las drogodependencias. De hecho, en la bibliografía sobre el tema aparecen solamente algunos programas aislados, principalmente en el ámbito estadounidense, que, sin embargo, muestran datos muy esperanzadores. Algunos ejemplos en este sentido son el Dade County Integrated Domestic Violence Model (Goldkamp, Weiland, Collins, 
y White, 1996) o el Yale Substance Abuse Treatment Unit's Substance Abuse-Domestic Violence Program (SATU-SADV) (Easton y Sinha, 2002).

Por lo que se refiere al programa desarrollado por Goldkamp et al. (1996), uno de los pioneros en este campo, los resultados obtenidos muestran cómo los pacientes que reciben una intervención focalizada a ambos problemas (adicción y violencia de pareja) obtienen mejores resultados y presentan una tasa más baja de reincidencia en las conductas violentas. Por su parte, el programa de Yale (SATU-SADV), basado en una intervención cognitivo-conductual con técnicas dirigidas al tratamiento de la adicción y al desarrollo de respuestas no agresivas hacia la pareja, muestra, como datos preliminares, una reducción importante tanto en el consumo de drogas como en los niveles de ira y de violencia hacia la pareja (Easton y Sinha, 2002). En un estudio más reciente de este grupo (Easton et al., 2007), llevado a cabo con 85 pacientes alcohólicos con problemas de violencia de pareja asociados, se comparó la eficacia de este programa de tratamiento cognitivo-conductual (SATU-SADV) con una intervención específica para el alcoholismo. Los pacientes que recibieron la intervención combinada mostraron un consumo de alcohol significativamente menor, así como una mayor reducción en la frecuencia de episodios violentos, en comparación con los que habían recibido la intervención estándar.

En cualquier caso, estos resultados, a pesar de ser claramente esperanzadores, no han sido todavía objeto de una evaluación rigurosa. Además, se trata de programas llevados a cabo fundamentalmente en el ámbito anglosajón, sin que existan hasta la fecha programas específicos desarrollados en nuestro país para la intervención simultánea con drogodependientes con problemas asociados de violencia contra la pareja. Ésta constituye, sin duda, una vía prometedora de estudio futuro.

\section{Programas basados en terapia de pareja}

La utilización de la terapia de pareja en el ámbito del maltrato hacia la mujer es un tema controvertido y no exento de polémica. Las situaciones de maltrato dentro de la pareja nunca se deben equiparar con los problemas de pareja que pueden afectar a cualquier relación afectiva. En nuestra opinión, la utilización de terapia de pareja no es una vía adecuada de intervención, ya que implícita o explícitamente distribuye la responsabilidad del maltrato a ambos miembros de la pareja. En los casos de violencia de género, la responsabilidad plena es de la persona que agrede, y nunca se debe compartir la misma con la víctima de dichas agresiones. Por ello, puede ser un error utilizar este tipo de estrategias para el tratamiento de los problemas de violencia de género. Es distinto, sin embargo, la utilización de sesiones terapéuticas específicas con ambos miembros de la pareja dentro de los programas de tratamiento de hombres agresores, habitualmente en fases avanzadas de la terapia y en casos con una evolución terapéutica razonable.

Sin embargo, a pesar de las críticas señaladas, algunos estudios estadounidenses (Chase, 0'Farrell, Murphy, Fals-
Stewart, y Murphy, 2003; Fals-Stewart, Kashdan, O'Farrell, y Birchler, 2002; O'Farrell, Van Hutton, y Murphy, 1999; O'Farrell, Murphy, Stephan, Fals-Stewart, y Murphy, 2004) han valorado la eficacia de la denominada terapia conductual de pareja para el tratamiento de la violencia de género en población drogodependiente. Uno de los principales estudios en este sentido es el desarrollado por O'Farrell et al. (2004), con una muestra de 303 hombres alcohólicos que participaron, junto con sus parejas, en un programa de terapia conductual de pareja. En esta investigación se utilizó también un grupo de control, compuesto también por 303 parejas, sin problemas de alcohol. Todas las parejas de la muestra recibieron una intervención conductual, basada en el programa desarrollado por el grupo de 0'Farrell y Fals-Stewart (O'Farrell y Fals-Stewart, 2000; 0'Farrell, et al., 2004). Se trata de un programa compuesto por 20-22 sesiones, de carácter semanal, aplicadas durante un período de entre 5 y 6 meses. Las 10-12 primeras sesiones se aplican con cada pareja en solitario (una hora de duración cada sesión), y las 10 restantes en un formato grupal con varias parejas simultáneamente ( 2 horas cada sesión). Los resultados obtenidos muestran que, en el grupo experimental, se produce una reducción significativa de las conductas violentas tras recibir la intervención terapéutica. En concreto, la tasa de conductas violentas contra la pareja disminuye del $60 \%$ antes de la intervención al 24\% después de la misma.

Estos resultados avalan los obtenidos en otros estudios previos de este mismo grupo (Fals-Stewart, et al., 2002), que muestran reducciones de las conductas violentas que oscilan desde tasas cercanas al 50\% antes de la intervención, a tasas del 17\% un año después de la terapia de pareja.

En resumen, los datos disponibles muestran la utilidad de los programas de pareja estudiados para el tratamiento conjunto de las conductas violentas contra la pareja en pacientes drogodependientes. Sin embargo, es necesario tener en cuenta que los resultados obtenidos en este sentido están avalados hasta la fecha por los estudios pertenecientes a un único grupo de investigación. Es necesario, por tanto, disponer de más datos para valorar la pertinencia y eficacia de este tipo de programas.

\section{CONCLUSIONES}

En este texto se ha presentado un análisis sobre la posible utilidad de aplicar programas de tratamiento de la violencia contra la pareja en contextos de atención a pacientes drogodependientes. Se trata de un tema novedoso que, hasta la fecha, ha sido muy poco estudiado. Las escasas investigaciones llevadas a cabo muestran que el maltrato a la pareja presenta una tasa de prevalencia elevada entre los pacientes adictos (Easton, et al., 2000; Fals-Stewart, et al., 2003). En la práctica clínica con drogodependientes es relativamente frecuente encontrar sujetos con órdenes judiciales de alejamiento de sus parejas. Tampoco es extraña la presencia de denuncias por malos tratos 0 , incluso, la existencia de antecedentes penales relacionados con conductas violentas. 
Como consecuencia de todo ello, algunos grupos de investigación (Easton, et al., 2007; Fals-Stewart y Kennedy, 2005; Goldkamp, et al., 1996; Moore, et al., 2008; Stuart, O'Farrell, Leonard, et al., 2009) han comenzado a estudiar la aplicación de programas conjuntos de tratamiento para pacientes adictos con conductas asociadas de maltrato a la pareja. En realidad, se trata de adaptar los programas de maltratadores existentes en la actualidad a este contexto específico de las conductas adictivas. Hoy en día, se cuenta con programas generales de intervención con maltratadores, así como con estudios que avalan la eficacia de los mismos (Babcock, Green, y Robie, 2004). La mayoría de estos estudios se han llevado a cabo con agresores que acuden a programas comunitarios de tratamiento (Boira y Jodrá, 2010; Echeburúa y Fernández-Montalvo, 1997; Echeburúa, Sarasua, Zubizarreta y Corral, 2009; Murphy, Taft, y Eckhardt, 2007), programas en prisión (Echeburúa, Fernández-Montalvo, y Amor, 2006; Echeburúa y Fernández-Montalvo, 2009) o programas para agresores derivados judicialmente (Eckhardt, Holtzworth-Munroe, Norlander, Sibley, y Cahill, 2008; Feder y Wilson, 2005). Muchos de estos programas no tienen en cuenta el consumo de sustancias y la mayoría de ellos excluye a los pacientes que presentan una conducta adictiva, y los deriva a programas específicos de intervención.

En este texto se ha puesto de manifiesto la frecuente presencia de violencia contra la pareja entre la población drogodependiente que, en muchas ocasiones, permanece oculta a lo largo del tratamiento (García, 2002; Stuart et al., 2009). Parece necesario, por tanto, contar con estudios que valoren la presencia de episodios de malos tratos hacia la pareja entre los pacientes adictos. Se trata de identificar la prevalencia de estas conductas y de establecer una descripción detallada de las características de estos pacientes. Asimismo, se necesita conocer la relación entre ambos aspectos $\mathrm{e}$, incluso, determinar su influencia en la permanencia o abandono de los programas de tratamiento de las drogodependencias.

En cualquier caso, en los estudios desarrollados se han puesto de manifiesto diferentes estrategias de tratamiento de la violencia contra la pareja entre los pacientes adictos. Por una parte, se ha comprobado que los programas estándar de tratamiento de las adicciones consiguen reducciones significativas de la violencia, a pesar de no llevar a cabo una intervención específica en este terreno. Por otra parte, se han desarrollado algunos programas específicos de intervención simultánea para ambas problemáticas. Los resultados obtenidos son todavía limitados y se centran en población anglosajona, sin que tengamos datos al respecto procedentes de nuestro pais. Sin embargo, son datos claramente esperanzadores y abren una línea de estudio novedosa y prometedora desde un punto de vista clínico.

En este sentido, algunas líneas futuras de investigación sobre esta problemática deben ir encaminadas, en primer lugar, al estudio de la tasa de prevalencia de agresores contra la pareja entre los usuarios de programas de tratamiento para adictos, así como de las características específicas de este tipo de pacientes. En segundo lugar, es necesario contar con investigaciones que establezcan la eficacia de programas de intervención psicológica destinados a pacientes drogodependientes que presentan conductas violentas en el hogar. Se trataría de adaptar los programas de intervención que ya han mostrado su utilidad para la eliminación y/o reducción de las conductas violentas y de las actitudes machistas en agresores contra la pareja, para ser aplicados de forma conjunta dentro de los programas de tratamiento de drogodependencias. Todo ello, supone sin duda, un reto importante para los próximos años.

\section{AGRADECIMIENTOS}

Este trabajo se ha financiado con la ayuda de un proyecto de investigación del Ministerio de Ciencia e Innovación (código PSI2009-08500).

\section{REFERENCIAS}

Babcock, J. C., Green, C. E., y Robie, C. (2004). Does batterer's treatment work? A metanalytic review of domestic violence treatment. Clinical Psychology Review, 23, 1023-1053.

Boira, S. y Jodrá, P. (2010). Psicopatología, características de la violencia y abandonos en programas para hombres violentos con la pareja: resultados en un dispositivo de intervención. Psicothema, 22, 593-599.

Chase, K. A., O'Farrell, T. J., Murphy, C. M., Fals-Stewart, W., y Murphy, M. (2003). Factors associated with partner violance among female alcoholic patients and their male partners. Journal of Studies on Alcohol and Drugs, 64, 137-149.

Easton, C. J., Mandel, D. L., Hunkele, K. A., Nich, C., Rounsaville, B. J., y Carroll, K. M. (2007). A cognitive behavioral therapy for alcohol-dependent domestic violence offenders: An integrated substance abuse-domestic violence treatment approach (SADV). American Journal on Addictions, 16, 24-31.

Easton, C. J., y Sinha, R. (2002). Treating the addicted male batterer: Promising directions for dual-focused programming. En C. Wekerle y A. Wall (Eds.), The violence and addiction equation: Theoretical and clinical issues in substance abuse and relationship violence (pp. 275-292). Nueva York: BrunnerRoutledge.

Easton, C. J., Swan, S., y Sinha, R. (2000). Prevalence of family violence entering substance abuse treatment. Journal of Substance Abuse Treatment, 18, 23-28.

Echeburúa, E., y Fernández-Montalvo, J. (1997). Tratamiento cognitivo-conductual de hombres violentos en el hogar: un estudio piloto. Análisis y Modificación de Conducta, 23, 355384.

Echeburúa, E., y Fernández-Montalvo, J. (2009). Evaluación de un programa de tratamiento en prisión de hombres condenados por violencia grave contra la pareja. International Journal of Clinical and Health Psychology, 9, 5-20.

Echeburúa, E., Fernández-Montalvo, J., y Amor, P. J. (2003). Psychopathological profile of men convicted of gender violence: A study in the prisons of Spain. Journal of Interpersonal Violence, 18, 798-812.

Echeburúa, E., Fernández-Montalvo, J., y Amor, P. J. (2006). Psychological treatment of men convicted of gender violence: $A$ 
pilot study in Spanish prisons. International Journal of Offender Therapy and Comparative Criminology, 50, 57-70.

Echeburúa, E., Sarasua, B., Zubizarreta, I. y Corral, P. (2009). Evaluación de la eficacia de un tratamiento cognitivo-conductual para hombres violentos contra la pareja en un marco comunitario: una experiencia de 10 años (1997-2007). International Journal of Clinical and Health Pschology, 9, 199-217.

Eckhardt, C., Holtzworth-Munroe, A., Norlander, B., Sibley, A., y Cahill, M. (2008). Readiness to Change, Partner Violence Subtypes, and Treatment Outcomes Among Men in Treatment for Partner Assault. Violence and Victims, 23, 446-475.

Fals-Stewart, W. (2003). The occurrence of partner physical aggression on days of alcohol consumption: A longitudinal diary study. Journal of Consulting and Clinical Psychology, 71, 41-52.

Fals-Stewart, W., Golden, J., y Schumacher, J. A. (2003). Intimate partner violence and substance use: A longitudinal day-to-day examination. Addictive Behaviors, 28, 1555-1574.

Fals-Stewart, W., Kashdan, T. B., O'Farrell, T. J., y Birchler, G. R. (2002). Behavioral couples therapy for drug-abusing patients: effects on partner violence. Journal of Substance Abuse Treatment, 22, 87-96.

Fals-Stewart, W., y Kennedy, C. (2005). Addressing intimate partner violence in substance-abuse treatment. Journal of Substance Abuse Treatment, 29, 5-17.

Fals-Stewart, W., Leonard, K. E., y Birchler, G. R. (2005). The occurrence of male-to-female intimate partner violence on days of men's drinking: The moderating effects of antisocial personality disorder. Journal of Consulting and Clinical Psychology, 73, 239-248.

Feder, L., y Wilson, D. B. (2005). A meta-analytic review of courtmandated batterer intervention programs: Can courts affect abusers'behavior? Journal of Experimental Criminlology, 1, 239-262.

Fernández-Montalvo, J., Echeburúa, E., y Amor, P. J. (2005). Aggressors against women in prison and in the community: An exploratory study of a differential profile. International Journal of Offender Therapy and Comparative Criminology, 49, 158-167.

Fernández-Montalvo, J., y Echeburúa, E. (1997). Variables psicopatológicas y distorsiones cognitivas de los maltratadores en el hogar: un análisis descriptivo. Análisis y Modificación de Conducta, 23, 151-180.

Fernández-Montalvo, J., y Echeburúa, E. (2005). Hombres condenados por violencia grave contra la pareja: un estudio psicopatológico. Análisis y Modificación de Conducta, 31, 451-475.

García, M.P. (2002). Alcohol y violencia familiar. Adicciones, 14, 3-8.

Goldkamp, J. S., Weiland, D., Collins, M., y White, M. (1996). The role of drugs and alcohol abuse in domestic violence and its treatment: Dade County Domestic Violence Court Experiment. Executive Summary of a Crime and Justice Research Institute Study: National Institute of Justice.

Klostermann, K. C., y Fals-Stewart, W. (2006). Intimate partner violence and alcohol use: Exploring the role of drinking in partner violence and its implications for intervention. Aggression and Violent Behavior, 11, 587-597.

Korman, L. M., Collins, J., Dutton, D., Dhayananthan, B., Littman-Sharp, N., y Skinner, W. (2008). Problem gambling and intimate partner violence. Journal of Gambling Studies, 24, 13-23.
Leonard, K. E. (2005). Alcohol and intimate partner violence: when can we say that heavy drinking is a contributing cause of violence? Addiction, 100, 422-425.

Moore, T. M., Stuart, G. L., Meehan, J. C., Rhatigan, D. L., Hellmuth, J. C., y Keen, S. M. (2008). Drug abuse and aggression between intimate partners: A meta-analytic review. Clinical Psychology Review, 28, 247-274.

Murphy, C. M., O'Farrell, T. J., Fals-Stewart, W., y Feehan, M. (2001). Correlates of intimate partner violence among male alcoholic patients. Journal of Consulting and Clinical Psychology, 69, 528-540.

Murphy, C. M., Taft, C. T., y Eckhardt, C. I. (2007). Anger Problem Profiles Among Partner Violent Men: Differences in Clinical Presentation and Treatment Outcome. Journal of Counseling Psychology, 54, 189-200.

Muñoz-Rivas, M., Gámez, M., Graña, J.L. y Fernández, L. (2010). Violencia en el noviazgo y consumo de alcohol y drogas ilegales en adolescentes y jóvenes españoles. Adicciones, 22, 125-134.

O'Farrell, T. J., y Fals-Stewart, W. (2000). Behavioral couples therapy for alcoholism and drug abuse. Journal of Substance Abuse Treatment, 18, 51-54.

O'Farrell, T. J., Fals-Stewart, W., Murphy, M., y Murphy, C. M. (2003). Partner violence before and after individually based alcoholism treatment for male alcoholic patients. Journal of Consulting and Clinical Psychology, 71, 92-102.

O'Farrell, T. J., y Murphy, C. M. (1995). Marital violence before and after alcoholism treatment. Journal of Consulting and Clinical Psychology, 63, 256-262.

O'Farrell, T. J., Murphy, C. M., Stephan, S. H., Fals-Stewart, W., y Murphy, M. (2004). Partner violence before and after couplesbased alcoholism treatment for male alcoholic patients: The role of treatment involvement and abstinence. Journal of Consulting and Clinical Psychology, 72, 202-217.

O'Farrell, T. J., Van Hutton, V., y Murphy, C. M. (1999). Domestic violence after alcoholism treatment: A two-year longitudinal study. Journal of Studies on Alcohol, 60, 317-321.

Stuart, G. L., O'Farrell, T. J., Leonard, K., Moore, T. M., Temple, J. R., Ramsey, S. E., et al. (2009). Examining the interface between substance misuse and intimate partner violence. Substance Abuse: Research and Treatment, 3, 25-29.

Stuart, G. L., O'Farrell, T. J., y Temple, J. R. (2009). Review of the association between treatment for substance misuse and reductions in intimate partner violence. Substance Use y Misuse, 49, 1298-1317.

Stuart, G. L., Ramsey, S. E., Moore, T. M., Kahler, C. W., Farrell, L. E., Recupero, P. R., et al. (2003). Reductions in marital violence following treatment for alcohol dependence. Journal of Interpersonal Violence, 18), 1113-1131.

Stuart, G. L., Temple, J. R., Follansbee, K. W., Bucossi, M. M., Hellmuth, J. C., y Moore, T. M. (2008). The role of drug use in a conceptual model of intimate partner violence in men and women arrested for domestic violence. Psychology of Addictive Behaviors, 22, $12-24$ 
